\title{
Association of lymphocyte to monocyte ratio and risk of in-hospital mortality in patients with acute type A aortic dissection
} \author{
Zhang $^{5}$, Fei Jiang ${ }^{2,3}$ \& Qiong Chen ${ }^{4}$ \\ ${ }^{1}$ Department of Nursing, Fujian Medical University Union Hospital, Fuzhou, PR China \\ ${ }^{2}$ Heart Medicine Research Center, Fujian Medical University Union Hospital, Fuzhou, PR China \\ ${ }^{3}$ Department of Cardiac Surgery, Fujian Medical University Union Hospital, Fuzhou, PR China \\ ${ }^{4}$ Department of Nursing, Fujian Medical University, Fuzhou, PR China \\ ${ }^{5}$ Department of Clinical Medicine, Fujian Medical University, Fuzhou, PR China \\ *Author for correspondence: fjxhyj|@163.com \\ $\ddagger$ Authors contributed equally
}

Yanjuan Lin ${ }^{*}, 1,2$, Yanchun Peng ${ }^{\ddagger 3,3}$, Yiping Chen ${ }^{4}$, Sailan $\mathrm{Li}^{2,3}$, Xizhen Huang ${ }^{2,3}$, Haoruo

\begin{abstract}
Aim: The aim of the study was to evaluate the relationship between lymphocyte to monocyte ratio (LMR) at admission and in-hospital mortality of patients with acute type $A$ aortic dissection (AAAD). Patients \& methods: We enrolled 536 patients with AAAD between June 2013 and December 2017. Patients were divided into two groups: the deceased group and the survival group. Results: In multivariable analysis, the association between LMR and in-hospital mortality was still significant. When the Q4 was set as the reference value, the odds ratios values of Q1, Q2 and Q3 were 4.4 (95\% Cl: 2.2-8.9; $\mathrm{p}<0.001), 1.4$ (95\% $\mathrm{Cl}: 1.1-3.4 ; \mathrm{p}=0.03)$ and $1.7(95 \% \mathrm{Cl}: 0.8-2.9 ; \mathrm{p}=0.158)$. Conclusion: Lower LMR may be independently associated with in-hospital mortality in AAAD.
\end{abstract}

First draft submitted: 14 November 2018; Accepted for publication: 14 August 2019; Published online: 4 October 2019

Keywords: acute type A aortic dissection $\bullet$ in-hospital outcomes $\bullet$ lymphocyte to monocyte ratio

Aortic dissection (AD) is a severe disease, in which the intima and/or medial dissection is expanded by high velocity blood flow, and the middle layer of artery is separated along the long axis to form a true and false lumen [1,2]. According to reports, the annual incidence of AD was 3.0-6.0 per 100,000 [3,4]. The occurrence rate of acute type A aortic dissection (AAAD) was 75\% [5], and AAAD is more severe and fatal than type $\mathrm{B} A \mathrm{AD}$. A study showed that the prognosis of untreated $\mathrm{AD}$ is extremely poor [6]. The mortality rate was $36-72 \%$ in the acute phase of $\mathrm{AD}$ in the hospital during $48 \mathrm{~h}$, an increase of $1-2 \%$ in the hourly mortality rate, and the mortality rate of 2 weeks was $89 \%$ [6]. According to the International Registry of Acute Aortic Dissection (AAD) the independent risk factors for $\mathrm{AD}$ mortality include age, hypotensive/shock, history of cardiac surgery and renal surgery, mesenteric or myocardial ischemia [7]. In addition, various biomarkers such as vascular injury, thrombosis and inflammation have been evaluated as contributors to AAD diagnosis or risk prediction tools [8]. In recent years, basic experiments and clinical studies have confirmed that the progression of $\mathrm{AD}$ has a significant correlation with the immune inflammatory response [9], and is related to poor prognosis of patients. White blood cells count and its subtypes are considered to be typical inflammatory biomarkers for predicting cardiovascular prognosis [10]. The neutrophil to lymphocyte ratio (NLR) has been assessed as a prognostic biomarker for various cardiovascular diseases [11]. In addition, the platelet to lymphocyte ratio (PLR) has predictive value for mortality and prognosis in AD patients [12].

lymphocyte to monocyte ratio (LMR), as a newly emerging inflammatory index, has potential value in predicting the severity and prognosis of coronary artery disease [13]. However, there is lack of data regarding the association of LMR with the in-hospital mortality in patients with AAD. We hypothesized that in-hospital mortality could be predicted using a novel inflammation biomarker, LMR, in patients admitted with AAAD. Therefore, we conducted an observational study to evaluate the impact of admission LMR on in-hospital mortality in patients with AAAD.

Future Medicine 


\section{Patients \& methods}

\section{Patients}

From June 2013 to December 2017, we enrolled 536 patients with AAAD by the Department of Cardiac Surgery of a Tertiary Care University Hospital. Patients were divided into two groups: the deceased group and the survival group. All patients were diagnosed as type A AD by computed tomographic angiography or magnetic resonance angiography [14]; age $\geq 18$ years old; drugs that affect blood cell counts such as aspirin, antibiotics and glucocorticoids have not been used; no history of malignant tumors; no previous history of cardiac surgery; no autoimmune disease; no chronic liver and kidney functional failure. The study was reviewed by the Ethics Committee of the hospital (ethical review no. 2013002).

\section{Data collection}

In the case of informed consent of the patient or family, trained undergraduate nursing staff performed data collection on patients with AAAD in the cardiac surgery ward or cardiac surgery ICU in strict accordance with the inclusion criteria. The patient's venous blood samples were obtained within $1 \mathrm{~h}$ after admission to hospital, and complete blood count was measured by Shenzhen Mindray BC-5800 blood cell analyzer. Including white blood cell counts, neutrophil counts, lymphocyte counts, monocyte counts and platelet counts. The LMR was calculated by dividing the absolute lymphocyte counts by the absolute monocyte counts. And vital signs were collected within $1 \mathrm{~h}$ of admission, including systolic blood pressure (SBP), diastolic blood pressure (DBP), pulse pressure (PP; the pressure difference between the systolic and diastolic pressures) and heart rates. At the same time, we evaluated all the demographic, including age, gender, medical history (hypertension, diabetes mellitus), smoking history and drinking history. Clinical data, including surgery, mechanical ventilation, the length of stay in ICU and the hospital stay, were also assessed. The primary end point of the study was hospital mortality, and the secondary end point was hospital complications, including renal insufficiency, liver insufficiency, pulmonary infection, arrhythmia, gastrointestinal hemorrhage, respiratory failure and multiple organ dysfunction syndrome (MODS).

\section{Definitions \& diagnostic criteria}

The AD was defined as patients have a typical history of chest pain, as well as evidence of computed tomographic angiography and magnetic resonance angiography. Stanford type A (DeBakey type I and type II) dissection involves the ascending aorta and/or aortic arch. Aches or other related symptoms occur within 2 weeks before admission, and $\mathrm{AAD}$ is defined as acute [14]. The smoking was defined as more than one cigarette/day for at least 6 months. The drinking was defined as those who reported consuming at least one drink/week for more than half a year. MODS was defined as a Sequential Organ Failure Score of 6 or more, on 2 or more consecutive days, at least $48 \mathrm{~h}$ after admission [15].

\section{Statistical analysis}

The data were analyzed using the Statistical Analysis System statistical software (version 9.4). Continuous variables with normal distributions are expressed as mean \pm standard deviation, while other variables are presented as median (25-75th percentile), categorical variables as number (percentage). Data were compared by the Student's $t$ test, Mann-Whitney U test, or $\chi^{2}$ test as appropriate. To evaluate the basic characteristics of patients who had a high LMR, we dichotomized the cohort with the median LMR (1.37) and compared characteristics between high and low LMR groups. The multivariate logistic regression analysis was performed to explore the relationship of LMR levels and lymphocyte and death in patients with AAAD. To assess the relationship between low LMR and lymphocyte and death in patients with AAAD, an adjusted logistic regression model 1 and model 2, an age, gender, AD rupture, HBP, WBC, neutrophil and surgery were used. To further analyze the relationship between LMR and death in patients with AAAD, and the quartiles of LMR levels were used as independent variables. Odds ratios (ORs) and 95\% CI were calculated. The comparison of the predictive value of mortality between the two groups was conducted by calculating the area under the curve using the receiver operating characteristic (ROC) curve. A p-value of $<0.05$ was considered statistically significant.

\section{Results}

From June 2013 to December 2017, a total of 581 patients with AAAD were enrolled. We excluded patients who met the following conditions: patients who had a history of cancer having a history of heart surgery, having a 


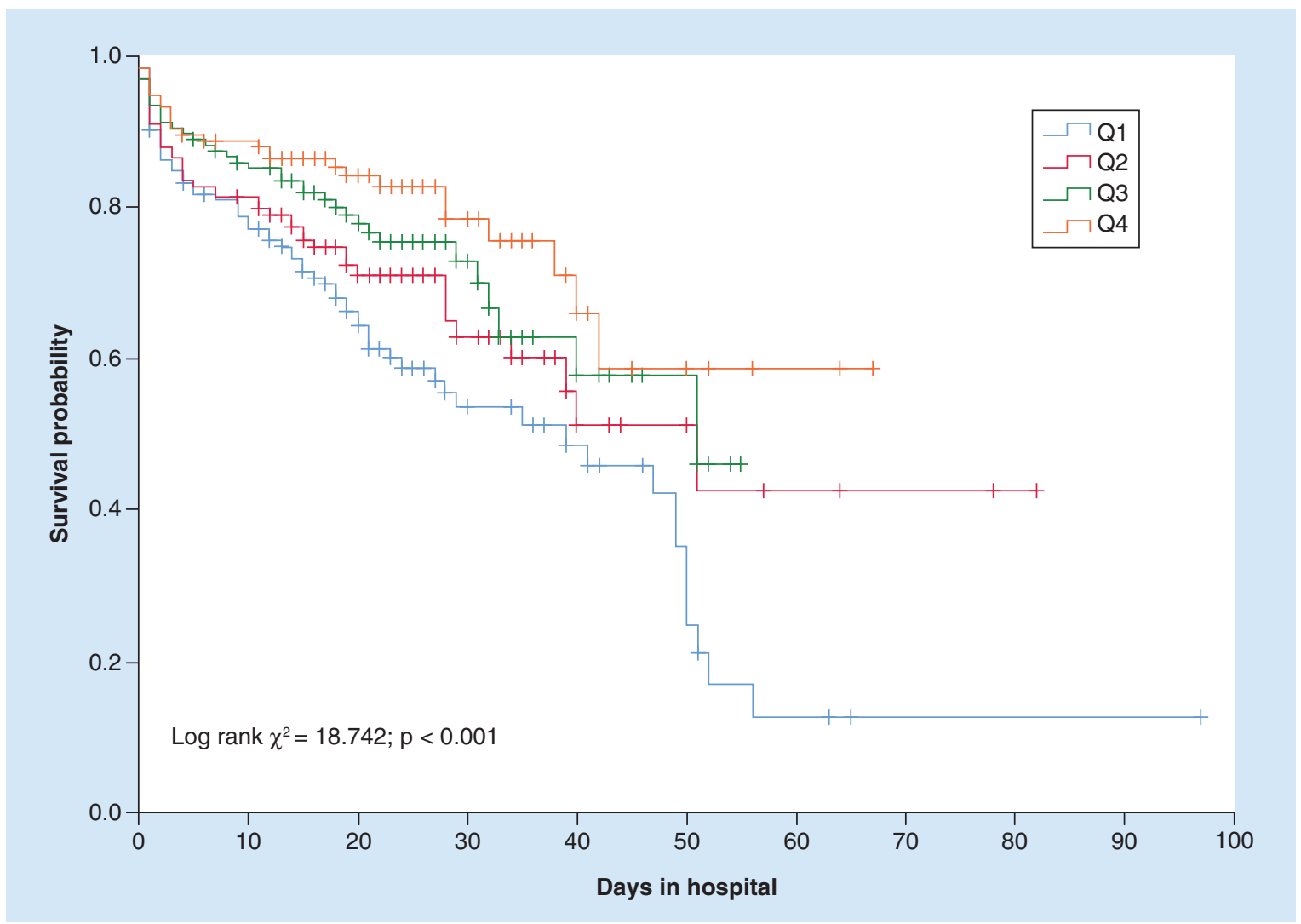

Figure 1. Kaplan-Meier survival curves for patients with acute aortic dissection were stratified by lymphocyte to monocyte ratio quartiles.

Q1: First quartile; Q2: Second quartile; Q3: Third quartile; Q4: Forth quartile.

history of severe renal disease, refused to take part in research or took a drug that affects the blood cell counts. After exclusion of 45 cases, 536 patients with AAAD were analyzed as two groups of patients, of whom 364 survived and 172 died. A total of 97 patients in this study did not undergo surgery. Total 66 patients had AD and died of hemorrhagic shock or pericardial tamponade on admission; 13 patients had ruptured $\mathrm{AD}$ while awaiting surgery, and all died during rescue. Total 18 patients have given up treatment for serious illnesses, and follow-up data showed that all of the patients passed away.

AAAD was divided into four groups according to the quartile of LMR of patients at hospital admission. The Kaplan-Meier survival curve of patients with AAAD according to the admission LMR level in quartiles shows a distinct bend duration of hospital stay toward lower mortality. Patients with lower LMR values had a higher mortality rate duration of hospital stay, as shown in Figure 1.

\section{Baseline characteristics of AAAD patients}

The baseline characteristics of AAAD patients are shown in Table 1. The age of the two groups were (52.94 \pm 11.83$)$ years old and $(57.03 \pm 12.26)$ years old, the difference was statistically significant $(\mathrm{p}<0.001)$. The proportion of males was higher than females, and the difference was statistically significant $(\mathrm{p}=0.018)$. There were no significant difference in smoker, drinker, hypertension, diabetes mellitus, SBP, DBP and PP ( $p>0.05)$. The difference were statistically significant in WBC, neutrophil, lymphocyte, NLR and LMR among the two groups $(\mathrm{p}<0.05)$. But there was no significant difference in monocyte $(\mathrm{p}=0.821)$. The median of platelet on survived is $168\left(\times 10^{9} / 1\right)$ and deceased is $157\left(\times 10^{9} / \mathrm{l}\right)$. There was significant difference between the two groups $(\mathrm{p}<0.005)$, as shown in Figure 2 . 
Table 1. Baseline characteristics of acute type A aortic dissection patients.

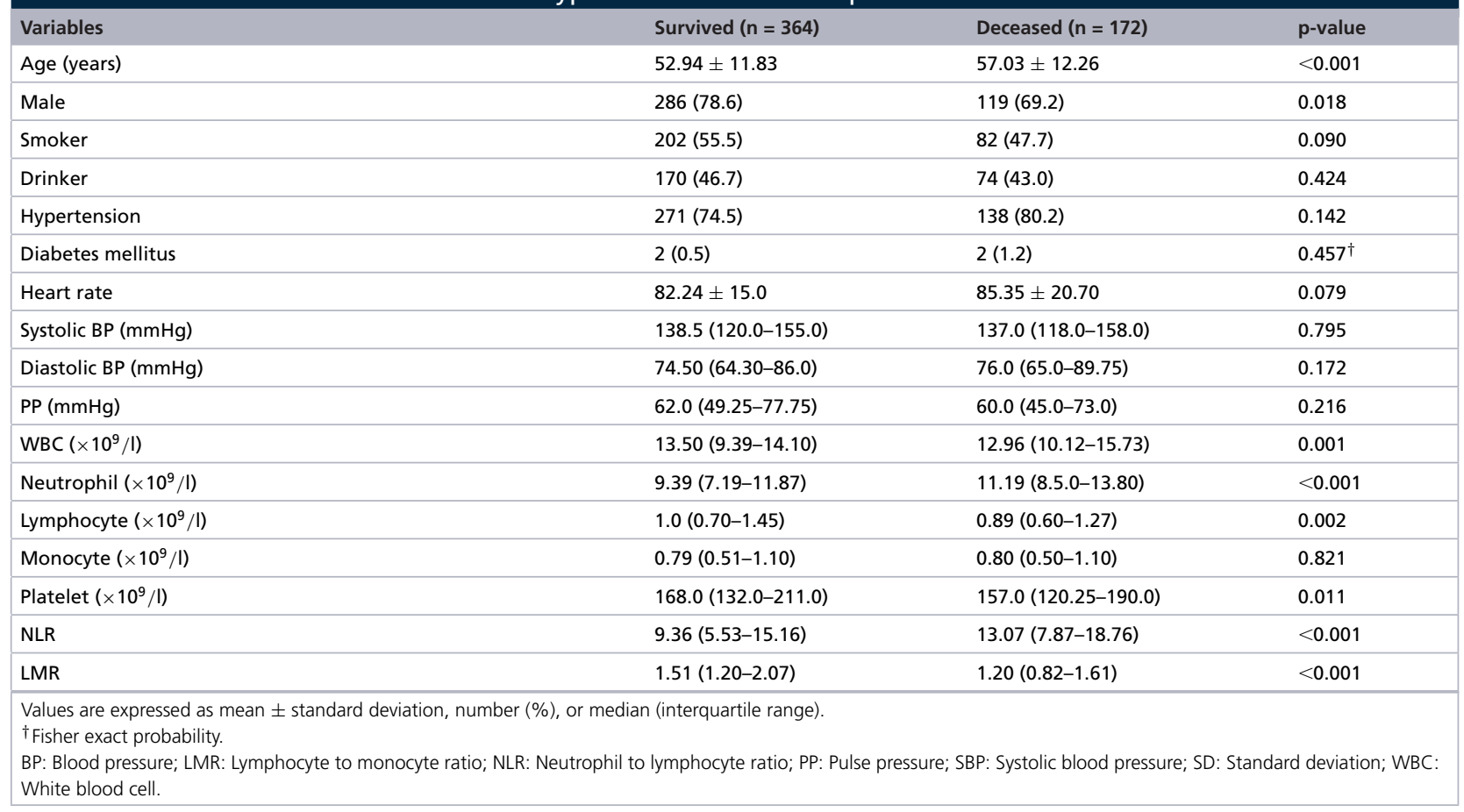

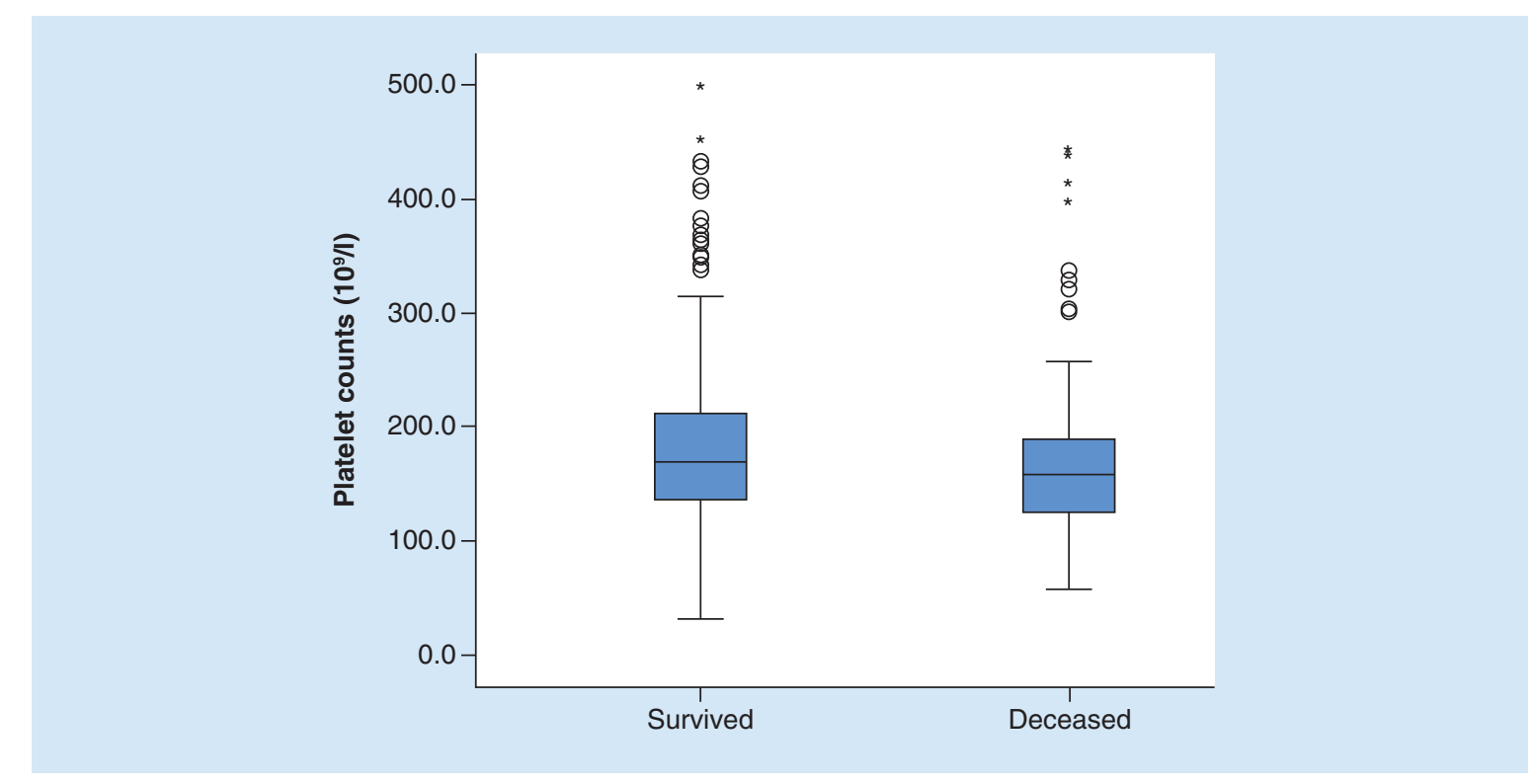

Figure 2. Platelet distribution in survived and deceased. The median of platelet on survived is $168\left(\times 10^{9} / \mathrm{l}\right)$ and deceased is $157\left(\times 10^{9} / \mathrm{l}\right)$. There was significant difference between the two groups $(p<0.005)$.

In-hospital events in patients with AAAD in different LMR groups

Patients were divided into low LMR $(<1.37)$ group and high LMR $(\geq 1.37)$ group according to the median LMR. Table 2 shows the in-hospital events in two groups of patients with AAAD. There were significant differences between the two groups in deceased, AD rupture, surgery, renal insufficiency and gastrointestinal hemorrhage $(\mathrm{p}<0.05)$. There was no significant difference in mechanical ventilation, cerebral infarction, arrhythmia, pulmonary infection, liver dysfunction, respiratory failure and MODS, ICU stay, and length of hospital stay between the two groups $(\mathrm{p}>0.05)$. 


\begin{tabular}{|c|c|c|c|}
\hline Variables & Low LMR; <1.37 $(n=267)$ & High LMR; $\geq 1.37(n=269)$ & $\mathrm{p}$-value \\
\hline Deceased & $109(40.8)$ & $63(23.4)$ & $<0.001$ \\
\hline Aortic dissection rupture & $43(16.1)$ & $25(9.3)$ & 0.018 \\
\hline Surgery & $206(77.2)$ & $233(86.6)$ & 0.004 \\
\hline Mechanical ventilation & $221(79.1)$ & $230(85.5)$ & 0.050 \\
\hline Renal insufficiency & $63(23.6)$ & $35(13.0)$ & 0.002 \\
\hline Liver insufficiency & $43(16.1)$ & $37(13.8)$ & 0.445 \\
\hline Pulmonary infection & $164(61.4)$ & $166(61.7)$ & 0.946 \\
\hline Arrhythmia & $5(1.9)$ & $3(1.1)$ & $0.467^{\dagger}$ \\
\hline Cerebral infarction & $17(6.4)$ & $12(4.3)$ & 0.329 \\
\hline Gastrointestinal hemorrhage & $33(12.4)$ & $11(4.1)$ & $<0.001$ \\
\hline Respiratory failure & $3(1.1)$ & $1(0.4)$ & $0.301^{\dagger}$ \\
\hline MODS & $7(2.6)$ & $4(1.5)$ & $0.351^{\dagger}$ \\
\hline ICU stay (days) & $5(3-10)$ & $4(3-7)$ & 0.210 \\
\hline Length of hospital stay (days) & $19(12-28)$ & $20(14-28)$ & 0.192 \\
\hline \multicolumn{4}{|c|}{$\begin{array}{l}\text { Values are expressed as mean } \pm \text { standard deviation, number (\%), or median (interquartile range). } \\
\begin{array}{l}\dagger \text { Fisher exact probability. } \\
\text { ICU: Intensive care unit; LMR: Lymphocyte to monocyte ratio; MODS: Multiple organ dysfunction }\end{array}\end{array}$} \\
\hline
\end{tabular}

\section{Table 3. Multivariate analysis of death in patients with acute type A aortic dissection.}

\begin{tabular}{|c|c|c|c|c|c|c|}
\hline \multirow[t]{2}{*}{ Variables } & \multicolumn{3}{|c|}{ Model 1} & \multicolumn{3}{|c|}{ Model 2} \\
\hline & OR & $95 \% \mathrm{Cl}$ & p-value & OR & $95 \% \mathrm{Cl}$ & p-value \\
\hline Age & 1.017 & $0.997-1.038$ & 0.096 & 1.016 & $0.996-1.036$ & 0.120 \\
\hline Gender (male) & 0.786 & $0.464-1.333$ & 0.372 & 0.754 & $0.446-1.275$ & 0.292 \\
\hline Aortic dissection rupture & 18.319 & $9.504-42.319$ & $<0.001$ & 18.517 & $9.237-41.170$ & $<0.001$ \\
\hline HBP & 1.815 & $0.987-3.339$ & 0.055 & 1.835 & $0.998-3.374$ & 0.051 \\
\hline WBC & 0.690 & $0.527-0.903$ & 0.007 & 0.845 & $0.621-1.149$ & 0.283 \\
\hline Neutrophil & 1.542 & $1.164-2.043$ & 0.003 & 1.273 & $0.924-1.754$ & 0.140 \\
\hline Surgery & 0.180 & $0.089-0.362$ & $<0.001$ & 0.165 & $0.082-0.333$ & $<0.001$ \\
\hline LMR (median) & 1.868 & $1.148-3.013$ & 0.012 & - & - & - \\
\hline Lymphocyte (median) & - & - & - & 1.648 & $0.924-3.069$ & 0.089 \\
\hline
\end{tabular}

HBP: High blood pressure; LMR: Lymphocyte to monocyte ratio; OR: Odds ratio; WBC: White blood count.

\section{Multivariate analysis for prediction of mortality in patients}

In multivariate regression analysis, it was found that LMR (AOR $=1.868 ; 95 \% \mathrm{CI}: 1.148-3.013 ; \mathrm{p}=0.012$ ) remained significant after adjusting for confounders (Table 3). The AD rupture (AOR = 18.319; 95\% CI: 9.50442.319; $\mathrm{p}<0.001)$, WBC (AOR = 0.690; 95\% CI: 0.527-0.903; p = 0.007), neutrophil (AOR = 1.542; 95\% CI: $1.164-2.043 ; \mathrm{p}=0.003)$, surgery $(\mathrm{AOR}=0.180 ; 95 \% \mathrm{CI}: 0.089-0.362 ; \mathrm{p}<0.001)$ were also significant, independent of LMR from model 1. But there were no statistically significant difference in age, gender, HBP ( $\mathrm{p}>0.05$ ). In model 2, replacing LMR with lymphocyte as a sensitivity analysis (Table 3 ). The lymphocyte was not statistically significant $(\mathrm{p}>0.05)$. The $\mathrm{AD}$ rupture $(\mathrm{AOR}=18.517 ; 95 \% \mathrm{CI}$ : 9.237-41.170; $\mathrm{p}<0.001)$, surgery $(\mathrm{AOR}=0.165 ; 95 \% \mathrm{CI}: 0.082-0.333 ; \mathrm{p}<0.001)$ were statistically significant. When the Q4 was set as the reference value, the OR values of Q1, Q2 and Q3 were 4.4 (95\% CI: 2.2-8.9; p < 0.001), 1.4 (95\% CI: 1.1-3.4; $\mathrm{p}=0.03)$ and $1.7(95 \% \mathrm{CI}: 0.8-2.9 ; \mathrm{p}=0.158)$ after adjusting for age, gender and hypertension in multiple factor logistic regression analysis, see Figure 3.

\section{The sensitivity \& specificity of prediction of death with LMR level}

According to the results of ROC curve, the AUC of the clinical variable ROC was 0.849 (95\% CI: 0.810-0.888; $\mathrm{p}<0.001$ ), the sensitivity was $79.1 \%$, and a specificity was $80.5 \%$. After adding LMR, the area under the ROC curve became 0.875 (95\% CI: 0.841-0.910; p < 0.001), with a sensitivity of $80.2 \%$ and a specificity of $81.0 \%$. 


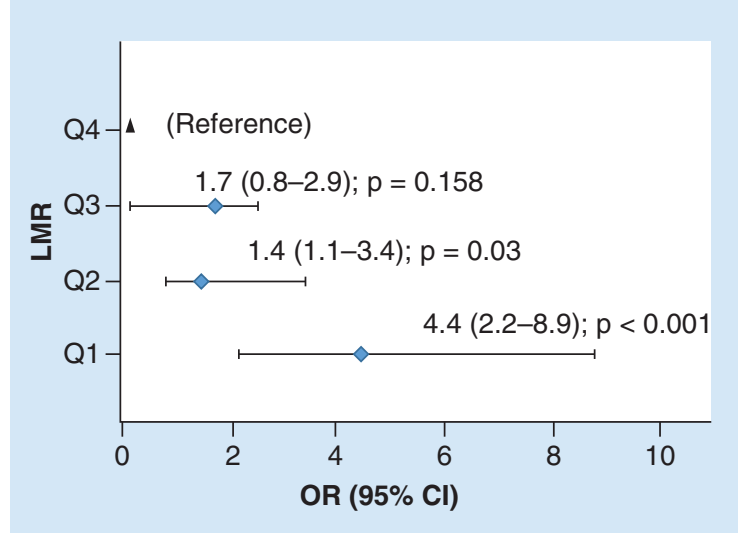

Figure 3. Relationship between different lymphocyte to monocyte ratio levels and in-hospital mortality in multiple factor logistic regression analysis. Adjustment: age, gender, hypertension, aortic dissection, mechanical ventilation, pulmonary infection, renal insufficiency, liver insufficiency, respiratory failure, gastrointestinal hemorrhage and multiple organ dysfunction syndrome. LMR: Lymphocyte to monocyte ratio; OR: Odds ratio; Q1: First quartile; Q2: Second quartile; Q3: Third quartile; Q4: Forth quartile.

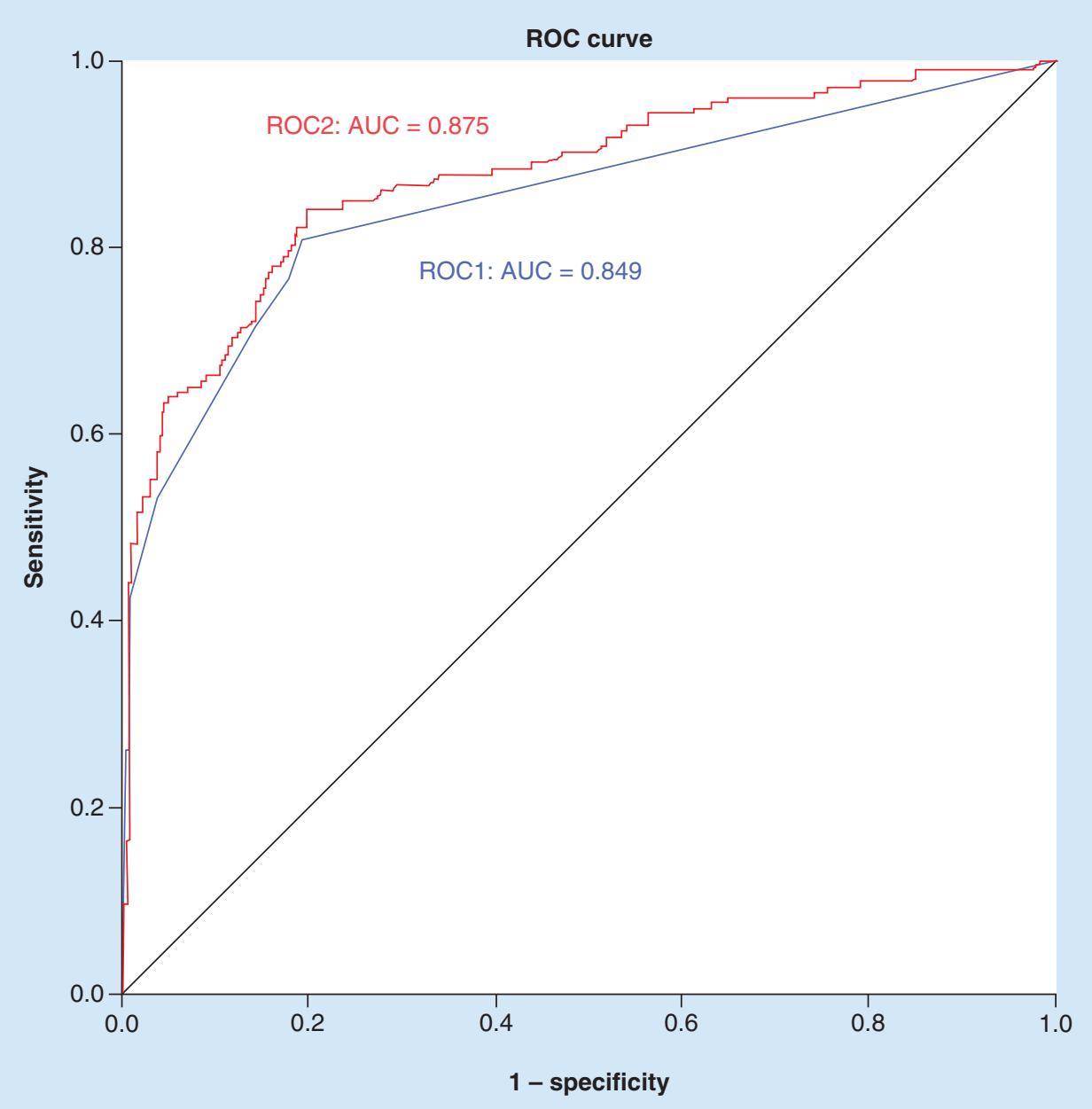

Figure 4. The receiver operating characteristic curves of predict death of patients with acute type $A$ aortic dissection. The difference between ROC1 and ROC2 is statistically significant $(p=0.002)$. ROC1 include aortic dissection rupture, mechanical ventilation, renal insufficiency, gastrointestinal hemorrhage. ROC2 include aortic dissection rupture, mechanical ventilation, renal insufficiency, gastrointestinal hemorrhage, lymphocyte to monocyte ratio. AUC: Area under curve; ROC: Receiving operating characteristic.

The difference in area under the two groups of curves was statistically significant $(\mathrm{p}=0.002)$, and the ROC curve was shown in Figure 4. 


\section{Discussion}

The present study, based on a large sample cohort of patients with AAAD, highlights the relevance of elevated LMR on admission with in-hospital death. Moreover, it also provided that decreased LMR is associated with an increased incidence of in-hospital mortality.

Medial degeneration is a major histologic feature of AD, consists of a depletion of smooth muscle cells, elastic fiber rupture and collagen degradation. In $\mathrm{AD}$, an imbalance has been found between the production of metalloproteases, which are able to degrade collagen and elastin fibres and MMP inhibitors [9,16]. Karakoyun et al. [17] have detected the presence of inflammatory cells in the aortic degeneration, including neutrophil, lymphocyte and monocyte, among others. Immune infiltrates made up of macrophages and activated $T$ lymphocytes have been found inside the media in aneurysms and dissections, even in the ascending thoracic aortic tract $[9,18]$. Monocytes in the blood accumulate in the intima and subintima of blood vessels. Under the action of some locally produced cytokines, monocytes differentiate into macrophages and start the inflammatory process. Moreover, it has been demonstrated that such lymphocytes are able to induce apoptosis of SMCs and synthesis of metalloproteases, and macrophages are able to release collagenase and elastase. Lymphocytes and macrophages, not only increase the expression of proteases and cell adhesion molecules but also release reactive oxygen species [9]. These cells also contribute to apoptosis of smooth muscle cells in the aortic artery, and finally lead to medial degradation. In addition, monocyte phenotypic regulation has become a specific therapeutic target for the prevention and treatment of cardiovascular disease [19]. Pamukcu et al. [20] showed that monocyte is closely related to the occurrence of acute coronary syndrome, and its high count at admission is closely related to the poor prognosis of acute coronary syndrome. Inflammatory activation of monocytes is accompanied by the release of various cytokines and an oxidative state that promotes oxidation of LDL and activation of endothelial cells [20].

However, the inflammatory response changes dynamically over time after the occurrence of dissection. After a transient neutrophil response, the patient with the worst prognosis showed significant and persistent lymphopenia. Studies have shown that lymphatic invasion of the dissected aorta has been associated with AAD rupture, and peripheral lymphocyte count significantly associated with lymphatic invasion [21]. In patients with acute myocardial infarction, the lower baseline lymphocyte count was associated with a higher risk of death in subsequent months [22,23]. Del Porto et al. [24] found a significant decrease in T lymphocytes in the peripheral blood of patients with $\mathrm{AD}$, and $\mathrm{B}$ lymphocytes were the opposite. Our study founded that peripheral blood lymphocyte counts decreased in patients with AAAD death. When acute $\mathrm{AD}$ occurs, induce a strong stress response in the body, promote the neurohumoral activation, release catenol amines and inflammatory mediators, and increased serum cortisol, which leads to the decrease of lymphocyte count, and a $\mathrm{CD} 4^{+} / \mathrm{CD}^{+} \mathrm{T}$ lymphocyte ratio inverted [23]. The relative reduction of lymphocyte count is considered to be anti-inflammatory, the negative index of heart healing and remodeling after myocardial infarction [25], and is closely related to poor clinical outcome [19]. Thus, the decrease of lymphocyte count may be related to the severity of inflammatory responses and neurohumoral activation.

Some inflammatory markers such as high-level WBC count, NLR, PLR, D-dimmer and CRP have been found to be independent risk factors for long-term mortality in $\mathrm{AD}[8,10]$, and they were associated with poor prognosis. Even after repair of the dissection, it remains elevated [26]. As an independent factor, LMR can predict adverse events of various cardiovascular diseases. Nunez et al. [27], they founded that lower lymphocyte counts and higher monocyte counts were associated with poorer cardiovascular outcomes. As the same time, the lower the LMR which mean a higher burden of inflammation. The exact mechanism of LMR in relation to the prognosis of AAAD patients remains unclear. Based on current evidence, lymphocytosis impairs the effectiveness of the immune system and is associated with poor outcomes in patients.

Gastrointestinal bleeding due to stress ulceration is the most commonly identified mechanism. Stress ulceration has been attributed to ischemia and/or reperfusion injury of the splanchnic territory and endotoxemia leading to the impairment of gastric and duodenal mucosal defense mechanisms. Our study found that there were statistically significant differences in gastrointestinal bleeding rate between the death group and the survival group. Sbarouni et al. [12] observed a decrease in platelet count in patients with type AAD, and our study was consistent with the study of both. The blood flow in the nonendothelial pseudocavity during the pathogenesis of the arterial dissection activates the coagulation system, with platelet activation, adhesion and thrombosis, leading to platelet depletion and bleeding tendency. The decrease of platelet count may reflect the consumption of platelet and may lead to bleeding tendency [28]. 
There have been reports of the risk factors for increased mortality in the hospital, such as age over 70, sudden chest pain, low blood pressure, acute kidney failure, abnormal electrocardiogram, nervous system dysfunction, aortic arch replacement and $\mathrm{d}$-dimer increase, among others [7]. In multivariate logistic regression analysis, this study founded that the dissection rupture, lower LMR, as independent predictors of in-hospital mortality. And age is a well-known $\mathrm{AD}$ mortality risk factors, but our research did not find it was associated with mortality, which may be the same reason as Karakoyun et al. [17], our patient population is also relatively young. The results of the logistic regression analysis showed that $\mathrm{OR}_{\mathrm{Q} 1}=4.4(95 \% \mathrm{CI}: 2.2-8.9 ; \mathrm{p}<0.001)$, indicating that the lower $\mathrm{LMR}$ was the higher risk factor for the death of AAAD. Furthermore, in our study, we found that using admission LMR level, $\mathrm{AD}$ rupture, mechanical ventilation, renal insufficiency, gastrointestinal hemorrhage predict in-hospital mortality with a sensitivity of $80.2 \%$ and a specificity of $81.0 \%$ in AAAD (AUC $=0.875 ; 95 \%$ CI: $0.631-0.789 ; \mathrm{p}<0.001$ ). It can be seen that $\mathrm{LMR}$ has a certain predictive value for the death of $\mathrm{AD}$ in hospital.

Several limitations of this study should be detailed. First, the study was a single center observational study, and larger sample size and multicenter trials are needed to further confirm the predictive value of LMR for AAAD. Second, we have tried to use the LMR to do the ROC curve, and the cut-off value is 1.43 , but the AUC does not reach 0.7. LMR has potential predictive value for the death of patients with AAAD. The strength of LMR alone is not enough, and needs to be combined with other indicators. Third, we recorded the complete blood counts at admission, but the changes in these markers at different time points were not recorded; a series of measurements at different time points during hospitalization might be more valuable for evaluation of the association between LMR and prognosis of AAAD. Fourth, we only analyzed the results of the patients in the hospital and did not follow-up after discharge.

\title{
Conclusion \& future perspective
}

The results of this study indicate that lower LMR levels at hospital admission is associated with higher mortality in patients with AAAD, and the prognostic effect is independent of other recognized risk factors. The LMR may be able to risk-stratify AAAD patients at admission for in-hospital mortality in the early phase. These results require validation in larger prospective studies.

\section{Summary points}

- Lymphocyte to monocyte ratio (LMR) has been suggested as indicators of the inflammatory process.

- Retrospective analysis of prospectively collected database.

- There was no significant difference in monocytes between the survival group and the decreased group.

- The predictive value of LMR is better than that of lymphocytes.

- Lower LMR levels at hospital admission is associated with higher mortality in patients with acute type A aortic dissection.

- LMR is predictive of clinical outcomes in patients with acute type A aortic dissection independent of other factors.

- Combined receiver operating characteristic based on multivariate regression analysis predicts high value of death.

\begin{abstract}
Author contributions
$Y$ Lin, $X$ Huang and F Jiang conceived the research and acquired research funding. S Li and Y Peng supervised the data collection and analysis. $Y$ Lin, $Y$ Peng and $Y$ Chen designed the data collection tools with input from other team members. Y Chen and Q Chen accepted recruitment of participants and managed the data. Y Peng and $\mathrm{H}$ Zhang conducted primary data analysis, with all other team members contributing to data interpretation. $Y$ Lin and $Y$ Peng contributed to manuscript preparation, and all authors contributed substantially to its revision. Y Lin takes responsibility for the paper as a whole.
\end{abstract}

Acknowledgments

The authors thank the Department of Cardiac Surgery of a tertiary care university hospital and Heart Medicine Research Center.

Financial \& competing interests disclosure

This work was supported by grants from the Fujian Medical Innovation Project (grant number 2012-CXB-17), National key specialty construction of clinical project and Facing the Rural grassroots promotion project Fujian Province (grant number 2014011). The authors have no other relevant affiliations or financial involvement with any organization or entity with a financial interest in or financial conflict with the subject matter or materials discussed in the manuscript apart from those disclosed.

The authors have no conflicts of interest to disclose. 


\section{Ethical conduct of research}

The study was reviewed by the ethics committee of the hospital (ethical review no. 2013002). All subjects or their families signed an informed consent form to participate in the study.

\section{Open access}

This work is licensed under the Attribution-NonCommercial-NoDerivatives 4.0 Unported License. To view a copy of this license, visit http://creativecommons.org/licenses/by-nc-nd/4.0/

\section{References}

Papers of special note have been highlighted as: • of interest

1. Ito T, Yasuda N, Kuroda Y, Sugawara M, Koyanagi T, Higami T. Acute gallbladder necrosis in a patient with acute type B aortic dissection. Ann. Vasc. Dis. 6(4), 748-750 (2013).

2. Isoda $\mathrm{S}$, Osako M, Kimura $\mathrm{T}$ et al. Coronary malperfusion due to flap suffocation after acute type A dissection surgery. Ann. Thorac. Cardiovasc. Surg. 18(2), 144-147 (2012).

3. Clouse WD, Hallett JW Jr, Schaff HV et al. Acute aortic dissection: population-based incidence compared with degenerative aortic aneurysm rupture. Mayo Clin. Proc. 79(2), 176-180 (2004).

4. Lu J, Lu Y, Wang X et al. Prevalence, awareness, treatment, and control of hypertension in China: data from 1.7 million adults in a population-based screening study (China PEACE Million Persons Project). Lancet 390(10112), 2549-2558 (2017).

5. Hagan PG, Nienaber CA, Isselbacher EM et al. The International Registry of Acute Aortic Dissection (IRAD): new insights into an old disease. JAMA 283(7), 897-903 (2000).

6. Pape LA, Awais M, Woznicki EM et al. Presentation, diagnosis, and outcomes of acute aortic dissection: 17-year trends from the International Registry of Acute Aortic Dissection. J. Am. Coll. Cardiol. 66(4), 350-358 (2015).

7. Rampoldi V, Trimarchi S, Eagle KA et al. Simple risk models to predict surgical mortality in acute type A aortic dissection: The International Registry of Acute Aortic Dissection Score. Ann. Thorac. Surg. 83(1), 55-61 (2007).

8. Kalkan ME, Kalkan AK, Gündeş A et al. Neutrophil to lymphocyte ratio: a novel marker for predicting hospital mortality of patients with acute type A aortic dissection. Perfusion 32(4), 321-327 (2017).

- Evaluate the relationship between admission neutrophil to lymphocyte ratio and hospital mortality in acute type $\mathrm{A}$ aortic dissection.

9. Nagareddy P, Smyth SS. Inflammation and thrombosis in cardiovascular disease. Curr. Opin. Hematol. 20(5), 457-463 (2013).

10. Fan X, Huang B, Lu H et al. Impact of admission white blood cell count on short- and long-term mortality in patients with type A acute aortic dissection: an observational study. Medicine 94(42), e1761 (2015).

11. Tamhane UU, Aneja S, Montgomery D, Rogers EK, Eagle KA, Gurm HS. Association between admission neutrophil to lymphocyte ratio and outcomes in patients with acute coronary syndrome. Am. J. Cardiol. 102(6), 653-657 (2008).

- Platelet to lymphocyte ratio is an inflammatory biomarker, which is significant for the diagnosis of type $A$ aortic dissection.

12. Sbarouni E, Georgiadou P, Kosmas E, Analitis A, Voudris V. Platelet to lymphocyte ratio in acute aortic dissection. J. Clin. Lab. Anal. 32(7), e22447 (2018).

13. Kose N, Akin F, Yildirim T, Ergun G, Altun I. The association between the lymphocyte-to-monocyte ratio and coronary artery disease severity in patients with stable coronary artery disease. Eur. Rev. Med. Pharmacol. Sci. 23(6), 2570-2575 (2019).

14. Erbel R, Aboyans V, Boileau C et al. ESC Committee for Practice Guidelines. 2014 ESC Guidelines on the diagnosis and treatment of aortic diseases: document covering acute and chronic aortic diseases of the thoracic and abdominal aorta of the adult. The Task Force for the Diagnosis and Treatment of Aortic Diseases of the European Society of Cardiology (ESC). Eur. Heart J. 35(41), 2873-2926 (2014).

15. Manson J, Cole E, De'Ath HD et al. Early changes within the lymphocyte population are associated with the development of multiple organ dysfunction syndrome in trauma patients. Crit. Care 20(1), 176 (2016).

16. Sakalihasan N, Limet R, Defawe OD. Abdominal aortic aneurysm. Lancet 365(9470), 1577-1589 (2005).

17. Karakoyun S, Gürsoy MO, Akgün T et al. Neutrophil-lymphocyte ratio may predict in-hospital mortality in patients with acute type A aortic dissection. Herz 40(4), 716-721 (2015).

18. He R, Guo DG, Estrera AL et al. Characterization of the inflammatory and apoptotic cells in the aortas of patients with ascending thoracic aortic aneurysms and dissections. J. Thorac. Cardiovasc. Surg. 131(3), 671-678 (2009).

19. Fan Z, Ji H, Li Y et al. Relationship between monocyte-to-lymphocyte ratio and coronary plaque vulnerability in patients with stable angina. Biomark. Med. 11(11), 979-990 (2017).

- Circulating monocyte to lymphocyte ratio level has potential in identifying the vulnerable plaques in the setting of stable angina.

20. Pamukcu B, Lip GY, Devitt A, Griffiths H, Shantsila E. The role of monocytes in atherosclerotic coronary artery disease. Ann. Med. 42(6), 394-403 (2010). 
21. Wu D, Choi JC, Sameri A et al. Inflammatory cell infiltrates in acute and chronic thoracic aortic dissection. Aorta 1(6), 259-267 (2013).

22. Dragu R, Khoury S, Zuckerman R et al. Predictive value of white blood cell subtypes for long-term outcome following myocardial infarction. Atherosclerosis 196(1), 405-412 (2008).

23. Núñez J, Núñez E, Bodí V et al. Usefulness of the neutrophil to lymphocyte ratio in predicting long-term mortality in ST segment elevation myocardial infarction. Am. J. Cardiol. 101(6), 747-752 (2008).

24. del Porto F, Proietta M, Tritapepe L et al. Inflammation and immune response in acute aortic dissection. Ann. Med. 42(8), 622-629 (2010).

- Circulating monocyte to lymphocyte ratio level has potential in identifying the vulnerable plaques in the setting of stable angina.

25. Núńez J, Núñez E, Sanchis J, Bodí V, Llàcer A. Prognostic value of leukocytosis in acute coronary syndromes: the Cinderella of the inflammatory markers. Curr. Med. Chem. 13(18), 2113-2118 (2006).

26. Schillinger M, Domanovits $\mathrm{H}$, Bayegan $\mathrm{K}$ et al. $\mathrm{C}$-reactive protein and mortality in patients with acute aortic disease. Intensive Care Med. 8(6), 740-745 (2002).

27. Núñez J, Miñana G, Bodí V et al. Low lymphocyte count and cardiovascular diseases. Curr. Med. Chem. 18(21), 3226-3333 (2011).

- The pathophysiological mechanisms that have been proposed for the observed association between low lymphocyte count and cardiovascular disease.

28. Gedik HS, Korkmaz K, Günertem OE et al. Relationship of admission neutrophil-to-lymphocyte ratio with in-hospital mortality in patients with acute type I aortic dissection. Turk. J. Med. Sci. 44(2), 186-192 (2014).

- Evaluate the relationship between admission neutrophil to lymphocyte ratio and in-hospital mortality in acute type I aortic dissection. 